\section{Automated protocol selection for energy efficient WSN applications}

C. Shen, S. Harte, E. Popovici, B. O’Flynn, R. Atkinson and A. Ruzzelli

A mature wireless sensor network automation tool requires the implementation of different protocols for different topology and application scenarios to optimise the overall system performance. Focusing on the transport layer energy-aware routing function for the Tyndall Heterogeneous Automated Wireless Sensor (THAWS), embedding a reactive routing protocol, an energy-efficient proactive heuristic protocol and a distributed geographical routing mechanism on the Tyndall 10 and $25 \mathrm{~mm}$ nodes is proposed. Those protocols manage small, medium and large sensor applications adaptively, while the end users of the THAWS tool are transparent from protocol selection.

Introduction: The Tyndall Heterogeneous Automated Wireless Sensors (THAWS) tool has two types of nodes with different node layers developed by the Tyndall National Institute [1]. Two modular nodes have been designed with a size of 10 by $10 \mathrm{~mm}$, and 25 by $25 \mathrm{~mm}$. These are referred to as the 10 and $25 \mathrm{~mm}$ nodes shown in Fig. 1. Sensor layers can then be connected with application-specific sensors. In addition to sensors, a battery or energy harvesting device can be connected to provide a power supply and each node can also provide its own energy level reading. The $25 \mathrm{~mm}$ node has an Atmel ATmega128 microcontroller with $128 \mathrm{kB}$ of program memory. It owns a layer using a Nordic nRF2401 transceiver and another layer using an Ember EM2420 ZigBee 802.15.4 compatible transceiver. There is also a $433 / 868 / 915 \mathrm{MHz}$ layer using a Nordic nRF905 transceiver, which allows a longer range. The $10 \mathrm{~mm}$ node is currently a single transceiver layer, which uses a Nordic nRF905 chip. The chip has a radio that is compatible with the Nordic nRF905 so this allows heterogeneous networks to be built.

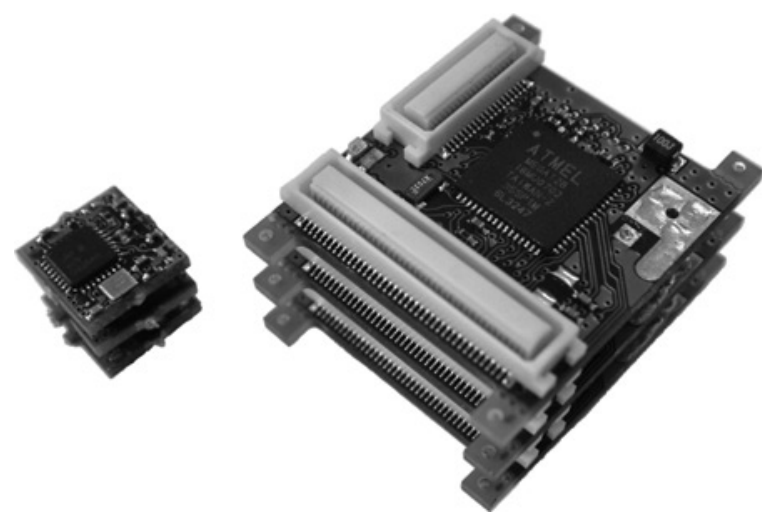

Fig. $110 \mathrm{~mm}$ (left) and $25 \mathrm{~mm}$ (right) modular Tyndall nodes

The core of the THAWS is an application generating tool, which has two main parts. The first part is a software library containing modules of codes that act as primitives in building up sensor applications, and the second part is a description of the desired application. The second step of developing the THAWS tool is to realise energy-aware functions focusing on dynamic routing as the key enabler. Instead of using only one specific protocol, we propose to take advantage of multiple protocols according to real-time system conditions and adaptively adjust the routing protocol based on real-time monitoring. Therefore, this Letter discusses a novel concept; the automated protocol selection for energy constrained wireless sensor networks (WSN).

Automated protocol selection: As fast deployment requires the interoperability between different components and interfaces, we first draw a simplified schematic for the automated network routing in Fig. 2 to introduce the main components, and input and output interfaces. Three categories of components are used for real-time sensor data monitoring, which are network topology, network representatives and application representatives. The network topology component monitors the changes of sensor node positions and sensor network topology when mobility is introduced to sensor nodes. The network representative periodically extracts the data to monitor the maximum delay of packets, network throughput and, most importantly, network energy efficiency. The application representatives also periodically monitor the feedback data from an application regarding the data of updated application throughput and stability.

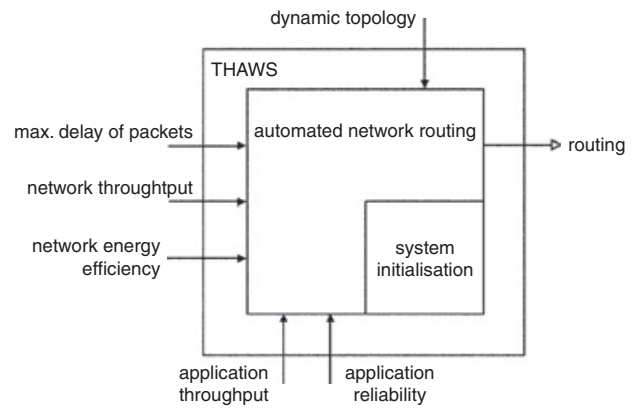

Fig. 2 Automated network routing components and interfaces schematic

The three components together provide the network input, while the input is translated into data context to be processed by the THAWS tool. The tool is located at a sensor network gateway, as such a design can provide IPv6 over low power wireless personal networks (6LowPan) based THAWS remote control, virtual control, and more importantly, both 25 and $10 \mathrm{~mm}$ nodes save energy on overhead data exchange. The gateway decides the best routing protocol and delivers the routing decision to nodes for different scenarios, e.g. city carbon footprint monitoring or an assisted living system. Three candidate routing protocols pre-stored in the nodes memory are: 1 . geographical routing (GR) [2], 2. proactive heuristic algorithm based routing with cost function (PR), and 3. reactive modified ad hoc on demand routing (RR). The PR is ideal for small and medium network topologies. A heuristic cost function can be used for further protocol optimisation or to emphasise the energy-aware objective

$$
F=\alpha E+\beta D+\gamma A
$$

Each parameter has been given a weight factor where $\alpha$ is for energy consumption of the nodes function $E, \beta$ is for packet delivery function $D$ and $\gamma$ is for the application data function $A$. The sum of $\alpha+\beta+\gamma=$ 1 and we can assign a bigger value to $\alpha$ to save node energy when finding the next hop node. The RR is suitable for large and dynamic network topologies, NST-AODV [3] is used to decrease data latency, increase system reliability and save power and memory storage costs. The GR applies on any network topologies with low mobility for efficient distributed data fusion applications. The protocol only requires each node to keep state only about its neighbours and does not require geographic location information owing to the 6LowPan implementation with a permanent IPv6 physical address.

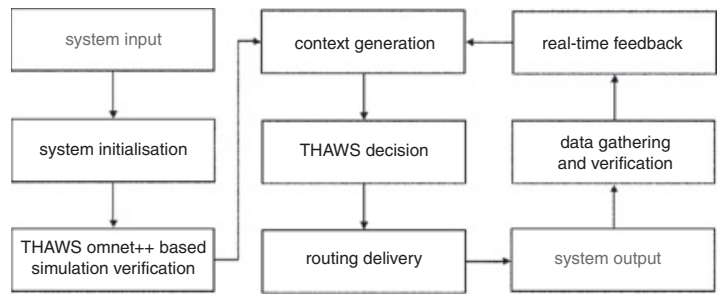

Fig. 3 THAWS automated routing operation flow

As shown in Fig. 3, the THAWS automated routing operates from the system input to the system output. The system initialisation block gathers information including network topology, user requirements, packet dissemination method, delay constraints, data format, etc., to feed the embedded OMNET $++[4]$ simulator. After the simulation verification, which lasts a few seconds, the results are automatically generated through a context block while the THAWS tool at a gateway makes the first routing protocol selection decision. The routing delivery continues with the operation flow. The THAWS tool then periodically collects real-time system output after the validation from the data feedback block. The application transporting method may migrate from one routing protocol to another based on the THAWS decision. 
The real-time online monitoring loop only ends when enough tasks have been finished or owing to system failure.

Discussion: The automated protocol selection method is the first to introduce protocol usage flexibility and self-configuration to an embedded sensor network. This real-time framework, before implementation, will be intensively tested and compared with other fixed protocol based systems to optimise system performance.

Conclusion: We propose a novel THAWS based automated routing selection method to fully utilise the system resource and save sensor system energy. The originality of the work is that the framework fully integrates the sensor network with 6LowPan, the protocol stack and the automated real-time feedback control and simulation based validation function. Self-management is achieved to overcome the rapidly growing sensor application complexity and to reduce the cost for operations.

(C) The Institution of Engineering and Technology 2009 29 April 2009

doi: $10.1049 / \mathrm{el} .2009 .1217$
C. Shen, S. Harte, E. Popovici and B. O'Flynn (CLARITY Research Centre, Tyndall National Institute, Cork, Ireland)

E-mail: vitoshen@gmail.com

R. Atkinson (Mobile Communications Group, University of Strathclyde, Glasgow, G1 1XQ, United Kingdom)

A. Ruzzelli (CLARITY Research Centre, University College Dublin, Dublin, Ireland)

\section{References}

1 Harte, S., Popovici, E., O’Flynn, B., and O’Mathuna, C.: 'THAWS automated design and deployment of heterogeneous wireless sensor networks', WSEAS Trans. Circuits Sys., 2008, 7, (9), pp. 829-838

2 Huang, H.: 'Adaptive algorithms to mitigate inefficiency in greedy geographical routing', IEEE Commun. Lett., 2006, 10, (3), pp. 150-152

3 Gomez, C., Salvatella, P., Alonso, O., and Paradells, J.: 'Adapting AODV for IEEE 802.15.4 mesh sensor networks: theoretical discussion and performance evaluation in a real environment'. Proc. IEEE WoWMoM, New York, USA, 2006

4 OMNET ++ discrete event simulator, http://www.omnetpp.org 